\title{
OBITUARY: Professor Ikuo Yasumasu
}

\author{
Professor Ikuo Yasumasu died on April 28, 2000 at the age of 70 from a heart attack. He had just \\ retired as Professor of Biology, School of Education, Waseda University.
}

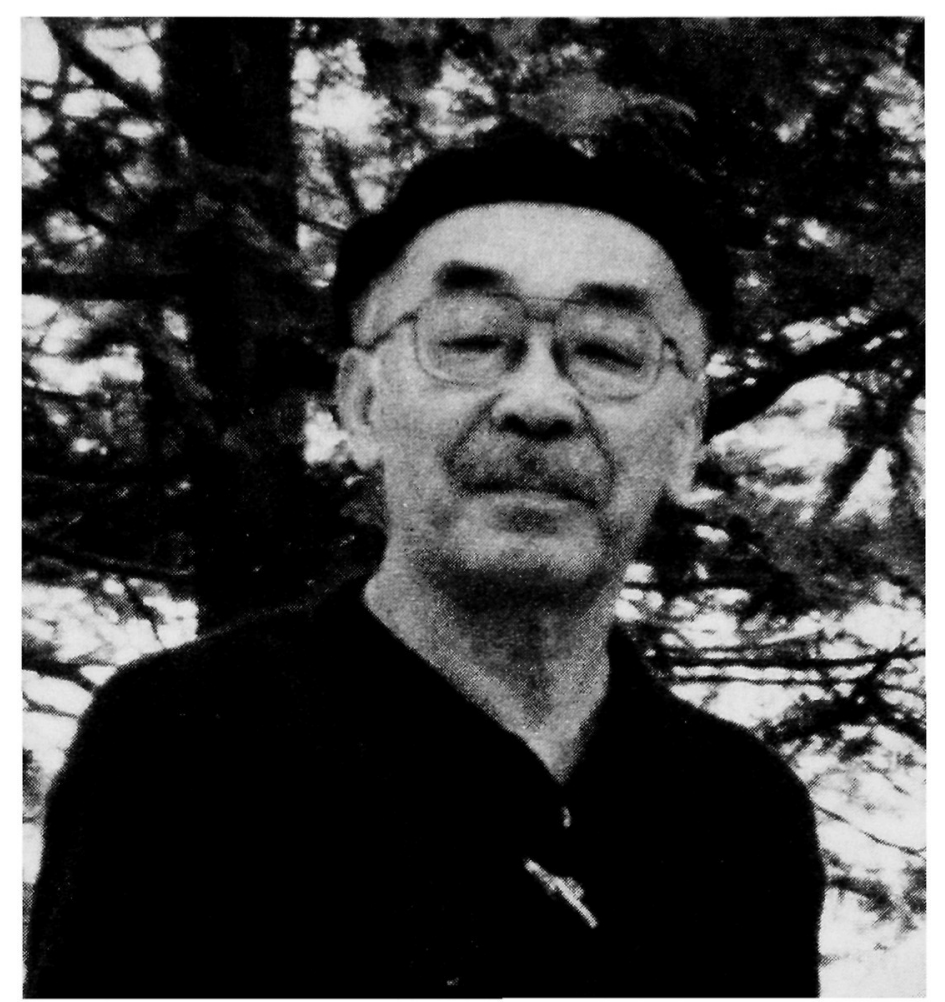

He was born in 1929 in Nagasaki, Japan. After graduating from the Zoological Institute, Faculty of Science, University of Tokyo, in 1955, he entered the graduate school of the University of Tokyo and began research in Professor Juro Ishida's laboratory. He earned the Doctor of Science degree in 1961 from the University of Tokyo with a dissertation topic concerning the sea urchin hatching enzyme and its crystallisation. From October 1960 to April 1966, he continued his research as an Instructor in the Department of Biology, Faculty of General Education, University of Tokyo. During this period he stayed eight months in the laboratory of Professor Alberto Monroy at Stazione Zoologica "Anton Dohrn" in Naples, Italy. In May 1966, he accepted a position as associate professor in Department of Biology, School of Education, Waseda University, and was promoted to full professor in 1971.

Starting from the crystallisation of the sea urchin hatching enzyme, which he did as a graduate student, he devoted much of his research activity to biochemical studies of sea urchin development from fertilisation to morphogenesis. However, he also published many articles on the biochemistry of hormone action and liver regeneration in mammals as well as articles on other topics of animal development including amphibian metamorphosis. Throughout his research career of nearly fifty years his interest was specially directed to glucose and lipid metabolism in eggs and embryos of sea urchins and other marine invertebrates, such as
Urechis, that provides energy for embryonic morphogenesis. Among his many and varied research efforts, he devoted many years to the elucidation of the mechanism underlying the sudden marked elevation of respiration that occurs just after fertilisation in sea urchin eggs, and at the time of hatching. Elevation of respiration after fertilisation in sea urchin eggs was first reported by Otto Warburg in the early 1900's, but remained unsolved since the initial observations of Warburg. Yasumasu finally determined that in unfertilised sea urchin eggs, electron transport is inhibited between flavoprotein and cytochrome c, and fertilisation somehow releases this inhibition, thereby elevating respiration. With these and related studies, he earned the Japan Zoological Society Prize in 1977. He continued his studies to find the mechanism underlying the marked increase in respiratory rate at hatching. In 1999 (Development Growth \& Differentiation 42: 155-165), he showed that the increase in respiration rate after hatching resulted from a decrease of ATP that regulated the acceptor control of respiration by ADP. In contemporary times, the major focus of developmental biologists is concentrated on understanding developmental phenomena at the level of gene expression. Therefore, little interest is focused on the study of respiration or oxidative phosphorylation where an adequate supply of ATP is obviously essential to drive the genetics of development. In this context, Yasumasu's accomplishments, though 
they attracted little attention at recent meetings of developmental biologists, significantly contributed to our fundamental understanding of animal development.

From 1986 to 1990 , he served as the President of the Japanese Society of Developmental Biologists (JSDB), and made great efforts to reduce the financial burden of the Society and to increase the circulation of the official international journal of JSDB, Development Growth \& Differentiation.

Yasumasu was a leader of sea urchin developmental biology in Japan for over 20 years, and many young scientists, as well as graduate students, in this research field were encouraged by his warm hearted but strict advice. With the purpose of encouraging young sea urchin developmental biologists, he held a conference on sea urchin developmental biology, the Akanezaki Symposium, every December from 1982 to 1998 in Akanezaki, Shizuoka, Japan; many research collaborations were encouraged by and resulted from this meet- ing. From December 9 to 11, 1999, he held The International Symposium on Fertilization and Development of Sea Urchin and Marine Invertebrates at the International Conference Hall of Waseda University, celebrating his 70th birthday. More than ten colleagues participated from abroad and many Japanese sea urchin biologists who were greatly indebted to Yasumasu for contributing to their scientific careers participated in the symposium.

Now to our sadness, Ikuo Yasumasu has gone away, and we cannot experience his enthusiastic discussions in a cafeteria around his lab on scientific topics reflecting his interests. We will miss him deeply.

\section{Hiraku Shimada}

Laboratory of Molecular Genetics

Graduate School of Science

Hiroshima University

Hiroshima

Japan 